\title{
A Framework for Tracking Former Patients in the Electronic Health Record Using an Educational Registry
}

\author{
Gregory E. Brisson, $M D^{7}$, Cynthia Barnard, $P h D, M B A, M S J S^{7}$, Patrick D. Tyler, $M D^{2}$, \\ David M. Liebovitz, $M D^{3}$, and Kathy Johnson Neely, $M D, M A^{7}$
}

${ }^{7}$ Northwestern University Feinberg School of Medicine, Chicago, IL, USA; ${ }^{2}$ Beth Israel Deaconess Medical Center, Boston, MA, USA; ${ }^{3}$ The University of Chicago Pritzker School of Medicine, Chicago, IL, USA.

One challenge of contemporary medical education is that shorter lengths of stay and time-limited clerkships often interrupt a student's relationship with a patient before a diagnosis is made or treatment is completed, limiting the learning experience. Medical students sometimes use electronic health records (EHRs) to overcome these limitations. EHRs provide access to patients' future medical records, enabling students to track former patients across care venues to audit their diagnostic impressions and observe outcomes. While this activity has potential to improve clinical training, there is a risk of unintended harm to patients through loss of privacy. Students need guidance on how to perform this activity appropriately. This article describes an ethical framework for tracking using an "educational registry," a list of former patients housed within the EHR that one follows longitudinally for educational purposes. Guiding principles include obtaining permission from patients, having legitimate educational intent, and restricting review of records to those essential for training. This framework could serve as a foundation for institutions seeking to develop a policy on tracking former patients, and may facilitate research on the use of EHRs to improve medical education, such as reducing diagnostic error and promoting self-directed learning.

KEYWORDS: medical education; electronic health records; medical ethics; patient privacy; legal issues in medicine.

J Gen Intern Med 33(4):563-6

DOI: $10.1007 / \mathrm{s} 11606-017-4278-5$

(c) Society of General Internal Medicine 2017

$\mathrm{M}$ ost medical students use electronic health records (EHRs), ${ }^{1,2}$ and there is consensus that educators should use them to improve training. ${ }^{3-7}$ One educational application of EHRs involves "tracking" patients, defined as following up on former patients in the EHR for educational purposes after they have left one's direct care. ${ }^{8}$ Tracking provides access to patients' future clinical data, enabling students to audit their

Received August 14, 2017

Revised October 27, 2017

Accepted December 14, 2017

Published online January 4, 2018 diagnostic impressions, ${ }^{5}$ observe outcomes, and develop deliberate practice patterns by offering feedback on questions such as, "Did we really need to order that?"7

Students find tracking educationally beneficial, ${ }^{9}$ but there is a risk to patient privacy associated with unlimited access to patient records. ${ }^{8,10}$ Horwitch has recommended that medical schools provide guidance on "how to do this that would not violate ethical or HIPAA requirements." 11

In this paper we describe a framework for tracking using an "educational registry" (EdR): a list of former patients housed within the EHR that one follows longitudinally for educational purposes. Our goal was to develop a tracking method that safeguards patient privacy while preserving the learning opportunities.

Registries are widely used in medicine, ${ }^{12-15}$ though little has been written about their use in medical education. An EdR is a tool for tracking the outcomes of patients one has encountered during training. At its most basic it is simply a list — but information contained within EdRs is HIPAA-compliant (as opposed to keeping a list on paper or a smartphone), and the term "registry" signifies that it is fundamentally different from patient lists used in daily clinical care. Below, we examine the rationale for EdRs and offer a framework for using them appropriately.

\section{RATIONALE FOR EDRS}

Medical students learn by observing disease patterns and treatment courses, a learning model which depends on longitudinal follow-up. ${ }^{16-20}$ However, time-limited clerkships and decreasing lengths of stay often interrupt a student's relationship with a patient before a diagnosis is made or treatment is completed, limiting the learning experience. ${ }^{20,21}$

One solution is the longitudinal clerkship model, which provides continuity not possible in traditional clerkships, though these are available to a minority of students. ${ }^{21-23}$ Another solution is the use of virtual patients that simulate longitudinal clinical experiences. ${ }^{24-26}$ However, these are expensive, and students lack an emotional connection to the subject which has been shown to improve learning. ${ }^{26,27}$

Educational registries offer benefits of both longitudinal clerkships and virtual patients. Hirsh et al. noted that, "In order to anchor clinical learning in caregiving, students must have 
relevant involvement with patients at the site and time of initial medical decision making, ideally before the diagnosis is made, and be able to follow patients for the duration of an illness episode (and beyond), ideally across care venues." 19 With EdRs, any patient encounter has potential to evolve into such a learning opportunity.

Alpert theorized that learners would benefit from a "secure institutional social media that delivers follow-up information to the trainee(s) involved." ${ }^{28}$ That is what an EdR does. And Dhaliwal and Detsky predict that master diagnosticians of the future will use the EHR to track outcomes, measure their diagnostic error rates, and regularly examine their mistakes. ${ }^{29}$ EdRs provide that capability. As McLaughlin and Coderre conclude, tracking "makes sense pedagogically."

\section{RISKS ASSOCIATED WITH TRACKING}

Tracking is not new. Hughes observes that students have always followed up on patients, "but that was usually through word of mouth." "With EHRs, ease of access to records - the very quality that facilitates tracking - greatly increases its potential for harm.

An ethical and legal analysis of tracking has been published, and a strong case can be made to permit tracking for education. ${ }^{8}$ However, students may underestimate the associated risk to privacy. ${ }^{10}$

What is difficult to appreciate about tracking is that it relates to the future. When patients grant the healthcare team access to their records, they understand that team members may see everything in their histories. What they may not realize is that, when tracking is allowed, they have unwittingly granted access to future events that may be completely unrelated to educational objectives. For example, students tracking a former patient with lupus might learn that this patient is treated for chlamydia at some later point. Despite assurances that students are bound by confidentiality, that new information is private, and the patient may simply not want students to know. $^{10}$

\section{A FRAMEWORK FOR USING EDRS}

Academic medical centers (AMCs) must assure patients that their records are being handled appropriately. ${ }^{30}$ Addressing this concern, we developed an ethical framework for tracking former patients. Essentially, the EdR is the tracking tool, and the framework is its instruction manual.

This framework was derived from widely accepted principles of medical ethics, ${ }^{31}$ the International Medical Informatics Association (IMIA) code of ethics, ${ }^{32}$ and the HIPAA Privacy Rule. ${ }^{33}$ It is also based on experience at our institution (GEB, $\mathrm{CB}, \mathrm{KJN}$ ), where $95 \%$ of users agree that it satisfies their obligation to respect patient privacy and preserve autonomy (unpublished data, 2017).

The framework is presented below.

\section{EdRs Must Be Treated with the Same Privacy and Security Protections as the EHR}

Information in the EHR acts as a patient-analogue, giving rise to its protected status. ${ }^{34}$ Information in an EdR is similarly protected, and the same requirements for maintaining confidentiality and privacy apply to records after the direct care relationship with the patient has concluded. Therefore, lists of former patients should be confined to the EHR.

\section{Students Should Ask Patients or Their Surrogates for Permission to Track Them}

Permission for tracking may be implicit in the general consent at AMCs, and tracking for educational purposes is permissible under the concept of "health care operations" described in the HIPAA Privacy Rule. ${ }^{33}$ However, compliance with laws and policies may not fully satisfy one's ethical obligation to patients.

Privacy can be defined as the ability for patients to control their personal information. ${ }^{35}$ If we accept as self-evident that patients have a right to privacy, then they have a fundamental right to control the use of their electronic records. ${ }^{32}$ Kluge has reasoned that, just as patients may "grant access to their bodies to individuals of their choice" (such as trainees), patients may "grant others permission to access their medical record." 36 Therefore, to safeguard privacy, explicit permission is optimal. ${ }^{8,11}$

Students should explain to patients that they would like to follow their progress through the EHR. The following script could be used to obtain permission: "It has been a pleasure taking care of you. If it's all right, I'd like to follow up in your medical record and see how you are doing. Would that be okay?" If patients object, students should not track them.

At our institution, we permit one student to obtain permission for other students who have had a meaningful clinical relationship (defined below) with that patient. This process minimizes the number of tracking requests patients receive.

\section{Students are Permitted to Track Only Those Patients with Whom They Have Had a Meaningful Clinical Relationship and Must Have Legitimate Educational Intent}

According to the IMIA, use of the protected health records must be "demonstrably necessary and not simply a matter of convenience." ${ }^{32,37}$ In addition, the HIPAA Privacy Rule requires hospitals to "restrict use of protected health information based upon the specific roles of the members of their workforce." ${ }^{33}$ Applied to EdRs, students must have a meaningful clinical relationship with a patient and legitimate educational intent to justify tracking.

What defines a "meaningful clinical relationship" is open to interpretation. We propose that it includes patients on whom students have performed a history and physical as a member of 
the primary service, performed a consult, or had significant cross-coverage. It also includes patients cared for by a service in which students were a part and with whom they had longitudinal contact, such as rounds, team discussions, and sign-out sessions.

Determining "legitimate educational intent" depends on the judgment of the student. Prior to using EdRs, students should receive training so they understand the privilege and responsibility associated with tracking. Just as we trust properly trained students to examine patients, we can trust properly trained students to examine the health records of former patients.

\section{Students Should Confine Their Review to the Minimum Information Necessary, and Are Permitted to Track Patients for the Minimum Length of Time Necessary to Achieve Educational Benefit}

According to the IMIA, access to the EHR must occur with a "minimum of interference with the rights of the affected person." ${ }^{32}$ Similarly, the HIPAA Privacy Rule states that use of the EHR is restricted to the "minimum amount of protected health information needed to accomplish the intended purpose." ${ }^{33}$ Applied to EdRs, students should confine their review of records to those which apply to their educational objective (i.e., follow-up on the condition for which they originally evaluated the patient) and should conclude when the educational objective has been achieved (resolution of the condition).

There is no standard for determining which health records are necessary for learning or when an educational objective has been achieved. One could argue that there is always something to be learned from follow-up. However, to respect patient privacy, one must have reasonable limits. With training on the rationale for such limits, students can be trusted to restrict their use of EdRs for education-recognizing that the duration of follow-up will vary depending upon the condition. For example, a patient with an allergic reaction that resolves in the hospital should not be tracked after discharge, but students may reasonably track a brain-injured patient through rehabilitation and recovery. Our experience suggests that students typically track patients from 1 to 6 months.

\section{Compliance Must Be Monitored}

The HIPAA Privacy Rule guarantees patients the right to an accounting of the disclosures of their electronic records. ${ }^{33}$ Applied to EdRs, EHR use for tracking must be monitored. To comply with HIPAA, AMCs routinely audit EHR use in their institutions, which should provide the necessary oversight of EdRs. Of note, since implementation at our institution, there have been no HIPAA violations related to EdR use (GEB, CB, KJN).
Guiding principles for using an educational registry (EdR)

- EdRs must be treated with the same privacy and security protections as the EHR.

- Students should ask patients or their surrogates for permission to track them.

- Students are permitted to track only those patients with whom they have had a meaningful clinical relationship and must have legitimate educational intent.

- Students should confine their review to the minimum information necessary, and are permitted to track patients for the minimum length of time necessary to achieve educational benefit.

- Compliance must be monitored.

\section{IMPLEMENTING EDRS AND FUTURE DIRECTIONS}

Many AMCs do not have a policy on tracking. ${ }^{8}$ This framework could serve as a foundation for institutions seeking to develop one.

Implementation of EdRs is straightforward: EHRs typically allow users to create custom patient lists. Tracking can be independent or incorporated into an existing curriculum, though students should receive training before starting. At our institution, students attend a class on EdRs and must have completed HIPAA training and a medical ethics course.

Currently, our students track independently. They are not required to track, though $96 \%$ report doing so. ${ }^{9}$ Ideally, students would be coached by faculty who meet with students to discuss their EdRs: which patients they enrolled in the registry, and why; how the permission conversation was conducted; and what they learned. However, EdRs are a new educational resource, and incorporating them into formal training will take time. In the interim, properly trained students should be permitted to track independently.

With a framework in place to guide tracking, research should focus on using EdRs to improve training. To date, the sole educational outcome that has been measured is the perceived utility of tracking by learners themselves. ${ }^{9}$ McLaughlin and Coderre observe that the clinical impact of tracking is "distant and dilute" and therefore difficult to measure, so they suggest studying proximal outcomes. ${ }^{20}$ Examples could include the impact of tracking on clinical reasoning, such as generating a differential diagnosis or using appropriate testing; or the impact on ethical decision-making, which may be strengthened, because tracking offers regular opportunities to manage ethical conflicts. EdRs may be particularly well-suited to measuring diagnostic error rates and improving the diagnostic process, described as a "professional imperative" by the Institute of Medicine. ${ }^{38}$ EdRs may even offer a novel opportunity to assess self-directed learning by monitoring EdR use to determine how often students track.

\section{CONCLUSION}

EdRs are a secure platform for tracking former patients for educational purposes. This article describes a framework to 
guide medical students on using them in an ethically appropriate manner. With training, students can begin using EdRs, and implementation should facilitate research on how to best use them to improve medical education.

\section{Acknowledgements:}

The authors wish to thank members of the Northwestern Memorial Hospital (NMH) Medical Ethics Committee for their spirited debate, which contributed to this framework. They would like to thank the members of the NMH Patient Family Advisory Council who generously participated in a focus group on the subject of this manuscript.

Prior Presentations: We have presented this topic at three conferences: Alliance for Academic Internal Medicine: "Innovating Medical Education: Updating Your Student Curriculum," in March, 2017; "The Patient, The Practitioner and the Computer: Holding on to the Core of Our Healing Professions in a Time of Technological Change," Warren Alpert School of Medicine of Brown University, March, 2017; and the American Society of Bioethics and Humanities national conference, October, 2017.

Corresponding Author: Gregory E. Brisson, MD; Northwestern University Feinberg School of Medicine, Chicago, IL, USA (e-mail: gbrisson@nm.org).

\section{Compliance with Ethical Standards:}

Conflict of Interest: The authors declare that they do not have a conflict of interest.

\section{REFERENCES}

1. Hammoud MM, Dalymple JL, Christner JG, et al. Medical student documentation in electronic health records: a collaborative statement from the Alliance for Clinical Education. Teach Learn Med. 2012;24(3):257-66.

2. http://www.aafp.org/news-now/education-professional-development/ 20130306med-students-ehrs.html. Accessed November 28, 2017.

3. Tierney MJ, Pageler NM, Kahana M, Pantaleoni JL, Longhurst CA. Medical education in the electronic medical record (EMR) era: benefits, challenges, and future directions. Acad Med. 2013;88(6):748-52.

4. Hammoud MM, Margo K, Christner JG, Fisher J, Fischer SH, Pangaro LN. Opportunities and challenges in integrating electronic health records into undergraduate medical education: a national survey of clerkship directors. Teach Learn Med. 2012;24(3):219-24.

5. Adibe BA, Jain SH. Electronic health records: potential to transform medical education. Am J Manag Care. 2010;16(12 Suppl HIT):SP62-3.

6. Peled JU, Sagher O, Morrow JB, Dobbie AE. Do electronic health records help or hinder medical education? PLoS Med. 2009;6(5):e1000069.

7. Stern RJ. Teaching Medical Students to Engage Meaningfully and Judiciously With Patient Data. JAMA Intern Med. 2016;176(9): 1397.

8. Brisson GE, Neely KJ, Tyler PD, Barnard C. Should Medical Students Track Former Patients in the Electronic Health Record? An Emerging Ethical Conflict. Acad Med. 2015;90(8):1020-4.

9. Brisson GE, Tyler PD. Medical Student Use of Electronic Health Records to Track Former Patients. JAMA Intern Med. 2016;176(9):1395-7.

10. Brisson GE, Neely KJ, Tyler PD, Barnard C. Privacy versus confidentiality: more on the use of the electronic health record for learning. Acad Med. 2015;90(8): 1001

11. Lawrence L. For med students, when does follow-up cross the line? 2017; https://acpinternist.acponline.org/archives/2017/03/medicaleducation.htm. Accessed November 28, 2017.

12. NIH Clinical Research Trials and You. http://www.nih.gov/health/ clinicaltrials/registries.htm. Accessed November 28, 2017.
13. Andersson K, Bray F, Arbyn M, et al. The interface of population-based cancer registries and biobanks in etiological and clinical research-current and future perspectives. Acta Oncol. 2010;49(8):1227-34.

14. Ortiz DD. Using a simple patient registry to improve your chronic disease care. Fam Pract Manag. 2006;13(4):47-8, 51-2.

15. AHRQ Health Information Technology: Computerized Disease Registries. http://healthit.ahrq.gov/key-topics/computerized-disease-registries. Accessed November 28, 2017.

16. Prober CG, Heath C. Lecture Halls without Lectures - A Proposal for Medical Education. N Engl J Med. 2012;366(18):1657-9.

17. Thistlethwaite JE, Davies D, Ekeocha S, et al. The effectiveness of case-based learning in health professional education. A BEME systematic review: BEME Guide No. 23. Med Teach. 2012;34(6):e421-44.

18. Norman G. Research in clinical reasoning: past history and current trends. Med Educ. 2005;39(4):418-27.

19. Hirsh DA, Ogur B, Thibault GE, Cox M. "Continuity" as an organizing principle for clinical education reform. N Engl J Med. 2007;356(8):858-66.

20. McLaughlin K, Coderre $\mathbf{S}$. Finding the middle path in tracking former patients in the electronic health record for the purpose of learning. Acad Med. 2015;90(8): 1007-9.

21. Norris TE, Schaad DC, DeWitt D, Ogur B, Hunt DD. Longitudinal integrated clerkships for medical students: an innovation adopted by medical schools in Australia, Canada, South Africa, and the United States. Acad Med. 2009;84(7):902-7.

22. Henschen BL, Garcia P, Jacobson B, et al. The patient centered medical home as curricular model: perceived impact of the "education-centered medical home". J Gen Intern Med. 2013;28(8):1105-9.

23. Worley P, Couper I, Strasser R, et al. A typology of longitudinal integrated clerkships. Med Educ. 2016;50(9):922-32.

24. Cook DA, Erwin PJ, Triola MM. Computerized virtual patients in health professions education: a systematic review and meta-analysis. Acad Med. 2010;85(10): 1589-1602.

25. Bateman J, Hariman C, Nassrally M. Virtual patients can be used to teach clinical reasoning. Clin Teach. 2012;9(2):133-4.

26. Ellaway R, Poulton T, Fors U, McGee JB, Albright S. Building a virtual patient commons. Med Teach. 2008;30(2):170-4.

27. Anderson AK, Wais PE, Gabrieli JD. Emotion enhances remembrance of neutral events past. Proc Natl Acad Sci U S A. 2006;103(5):1599-1604.

28. Alpert JS. Required Reading for Anyone Involved in Postgraduate Medical Education (Part 2). Am J Med. 2015;128(9):929-30.

29. Dhaliwal G, Detsky AS. The evolution of the master diagnostician. JAMA. 2013;310(6):579-80.

30. Miron-Shatz T, Elwyn G. To serve and protect? Electronic health records pose challenges for privacy, autonomy and person-centered medicine. Int J Pers Cent Med. 2011;1(2):405-9.

31. Beauchamp TL, Childress JF. Principles of Biomedical Ethics. 7th Edition ed. New York: Oxford University Press; 2009.

32. IMIA Code of Ethics for Health Information Professionals. http://imiamedinfo.org/wp/wp-content/uploads/2015/07/IMIA-Code-of-Ethics2016.pdf. Accessed November 28, 2017.

33. Summary of the HIPAA Privacy Rule. 2013; https://www.hhs.gov/hipaa/ for-professionals/privacy/laws-regulations/index.html. Accessed November 28, 2017.

34. Kluge EH. Security and privacy of EHR systems - ethical, social and legal requirements. Stud Health Technol Inform. 2003;96:121-127.

35. Winkelstein P. Ethical and Social Challenges of Electronic Health Information. In: Chen H, Fuller SS, C. F, Hersh W, eds. Medical Informatics: Knowledge Management and Data Mining in Biomedicine: Springer; 2005: 144-145.

36. Kluge EH. The Ethics of Electronic Patient Records. New York: Peter Lang Publishing: 2001.

37. Kluge EH. Informed consent to the secondary use of EHRs: informatic rights and their limitations. Stud Health Technol Inform. 2004;107(Pt 1):635-8.

38. Committee on Diagnostic Error in Health Care, Board on Health Care Services, Institute of Medicine, The National Academies of Sciences, Engineering, and Medicine; Balogh EP, Miller BT, Ball JR, eds. Improving Diagnosis in Health Care. Washington (DC): National Academies Press (US); 2015. 